\title{
Older Adults' Perceptions of Clinical Fall Prevention Programs: A Qualitative Study
}

\author{
Rebecca Calhoun, ${ }^{1}$ Hendrika Meischke, ${ }^{1}$ Kristen Hammerback, ${ }^{1}$ Alex Bohl, ${ }^{1}$ \\ Pamela Poe, ${ }^{2,3}$ Barbara Williams, ${ }^{1}$ and Elizabeth A. Phelan ${ }^{1,4}$ \\ ${ }^{1}$ Department of Health Services, School of Public Health, University of Washington, Seattle, WA 98195, USA \\ ${ }^{2}$ Department of Humanities and Communication Arts, Cheyney University of Pennsylvania, Cheyney, PA 19319, USA \\ ${ }^{3}$ School of Nursing and Health Sciences, La Salle University, Philadelphia, PA 19141, USA \\ ${ }^{4}$ Division of Gerontology and Geriatric Medicine, Department of Medicine, University of Washington, 325 9th Avenue, \\ Box 359755, Seattle, WA 98104, USA
}

Correspondence should be addressed to Elizabeth A. Phelan, phelane@uw.edu

Received 5 January 2011; Accepted 15 March 2011

Academic Editor: Steven Hooker

Copyright (C) 2011 Rebecca Calhoun et al. This is an open access article distributed under the Creative Commons Attribution License, which permits unrestricted use, distribution, and reproduction in any medium, provided the original work is properly cited.

\begin{abstract}
Objective. To investigate motivational factors and barriers to participating in fall risk assessment and management programs among diverse, low-income, community-dwelling older adults who had experienced a fall. Methods. Face-to-face interviews with 20 elderly who had accepted and 19 who had not accepted an invitation to an assessment by one of two fall prevention programs. Interviews covered healthy aging, core values, attributions/consequences of the fall, and barriers/benefits of fall prevention strategies and programs. Results. Joiners and nonjoiners of fall prevention programs were similar in their experience of loss associated with aging, core values they expressed, and emotional response to falling. One difference was that those who participated endorsed that they "needed" the program, while those who did not participate expressed a lack of need. Conclusions. Interventions targeted at a high-risk group need to address individual beliefs as well as structural and social factors (transportation issues, social networks) to enhance participation.
\end{abstract}

\section{Background}

Falls are common among older adults [1], and their consequences often devastating and costly [2-4]. Evidencebased interventions are available to prevent falls [5-7]; however, we have observed that care-seeking by older adults to reduce their likelihood of falling is less than expected. Others have also observed that few older adults engage in proven behaviors to reduce fall risk after a fall [8-11]. Explanations identified through qualitative research with older persons have included an underestimation of personal susceptibility to falling [11], a sense of fatalism or a belief that falls occur due to bad luck [12], an attribution of falls to external (rather than within-person) causes [12], a belief that falls are accidental [13], a belief that falls are an inevitable consequence of aging [12], and a belief that one already knows what to do to prevent falls [13].
The majority of published research has been conducted with persons from Scandinavia, Britain, New Zealand, and Australia $[10,11,13]$, and thus it is unknown whether the explanations for this phenomenon, as observed in these studies, are generalizable to elders residing in the United States. Additionally, most research has been conducted with samples that have not been fully characterized in terms of educational level and socioeconomic status [10-13] and with persons who may or may not have experienced a fall $[10,11]$. Thus, it is also unclear whether the findings apply to elders of different ethnic backgrounds, to low-income elders, or to elders at high risk of falls. An understanding of contextual (personal, environmental) factors [14] affecting program participation is important, as program participation may ultimately influence an individual's likelihood of future falls and fall-related injuries, global functioning, and quality of life [2-4]. We, therefore, conducted the present study with 
a diverse, low-income, community-dwelling sample that had experienced a fall to better understand the lower than anticipated uptake of clinical fall prevention services by older Americans.

\section{Materials and Methods}

2.1. Study Design. We hypothesized that an array of complex factors would affect the decision to participate in a clinically oriented, fall prevention program and that beliefs, attitudes, and knowledge would differ between those who were receptive to a fall prevention service and those who were not. We conducted in-depth qualitative interviews of elders who had experienced a fall to explore differences between fall prevention program "joiners" and "nonjoiners." We employed a purposeful stratified sampling strategy to ensure an adequate balance of joiners and nonjoiners [15]. We use the terms "join" and "participate" interchangeably for those accepting an invitation to receive a fall risk assessment and management recommendations from either of two fall prevention programs.

\subsection{Data Collection}

2.2.1. Setting. Interviews were conducted between February and August 2008, in King County, Washington. All participants had previously been referred to one of two Seattle-based fall prevention programs: Harborview Medical Center's Fall Prevention Clinic or King County Emergency Medical Services (EMS) Fall Prevention Program, "One Step Ahead." The Harborview program is a hospital-based, outpatient clinic that offers multifactorial assessment and longitudinal management of modifiable fall and fall injury risk factors to persons with a history of falls [16]. The King County EMS program is a home-based program in which a physical therapist conducts a home safety assessment and arranges for any recommended adaptive devices to be installed. The therapist also assesses balance, strength, and footwear and reviews medical conditions and medications in order to customize a personal action plan. Upon written approval of the participant, the therapist sends a letter to the personal physician explaining the "One Step Ahead" program and detailing findings of the evaluation so that the physician may consider if other medical management might also be beneficial to prevent future falls. More information about the EMS program is available at http://www.kingcounty.gov/ healthservices/health/ems/community/falls.aspx.

2.2.2. Recruitment. Participants were recruited from lists provided by the two programs. A representative of each program sent letters to potential participants with information about the study, an invitation to participate, and a phone number to call for further information or to schedule an interview. In addition, each mailing included a stamped postcard for potential participants to return if they preferred the option of receiving a phone call from an interviewer or if they wished to decline participation. A follow-up phone call was made to those potential participants who neither called nor returned a postcard within a few weeks of receiving the mailing. For each program, recruitment targeted an equal number of participants who had accepted and joined the program and those who had either declined to join or otherwise not followed up on an invitation or referral. Men and women aged 50 and above were recruited in proportion to their representation in the population. Other eligibility criteria included residing in King County, English fluency, and possessing the cognitive ability to clearly understand the decision to participate in the study.

2.2.3. Interview Instrument. The interview guide consisted of a series of open-ended questions designed to facilitate a conversation during which individual motivators and barriers to participation in the specified programs might be identified and discussed. The first half of the interview covered the role of health-related beliefs and behavior in participants' daily lives, particularly as it related to views on healthy aging and participants' core values. The second half focused specifically on attitudes and behaviors surrounding fall prevention, including participants' experiences of their own falls and efforts to prevent falling again. Interview questions were developed by the investigators to address the phenomenon of interest (i.e., lower than anticipated participation in available clinical services to address fall risk on the part of older adults with a history of having fallen). The first author (EAP) was familiar with the small body of relevant literature on the topic; however, no particular preconceived hypotheses were explored via the interview questions, nor were the interview questions designed to confirm or disprove findings from other published work.

2.2.4. Interview Procedure. All interviews were conducted by research assistants who received training in conducting semistructured, in-person interviews. Interviewers scheduled appointments by phone and conducted the interviews at participants' homes or at another location of the participant's choosing. All interviews were audiotaped, and an incentive to participate was provided in the form of a gift card worth $\$ 25$. Each interview lasted approximately 45 minutes, and upon conclusion, participants completed a paper questionnaire of standard demographic information about themselves and their households. Throughout the process, interviewers were monitored to ensure both quality control and interinterviewer consistency.

All study procedures were approved by the Institutional Review Board at the University of Washington.

2.3. Analysis. Each interview was transcribed verbatim by a professional transcriptionist. Transcripts were then uploaded into Atlas ti version 5.2 (Scientific Software Development $\mathrm{GmbH}$, Berlin, Germany), a computer software program that facilitates qualitative data analysis. Interviews were grouped based on the participant's status as either a program "joiner" or "non-joiner," as the primary focus of this study was to ascertain differences between these two groups.

Coding and theme development was guided by the grounded theory approach of Strauss and Corbin $[17,18]$. This methodology was chosen because our main purpose was 
to arrive at an understanding of people's actions/behaviors with regard to care-seeking for falls/fall risk. All coding was completed by two coders with experience in qualitative coding and analysis, working independently. The two coders and a third research team member read through all interviews prior to coding and theme development to establish initial impressions and to facilitate grounding in the data. An initial start list of codes [15] was developed by identifying a priori areas of interest, such as "consequences of falling" and "barriers to joining," and was included in the interview guide. The second stage of code development consisted of line-by-line reading of the interviews to capture subcodes that emerged from the data. These sub-codes were organized under the start list codes developed in the first stage. In this initial coding stage, an extensive codebook was developed that included definitions for the start list and all related sub-codes. Coding discrepancies were reviewed with the full research team until consensus on final codes was reached. Because coding was done by consensus, interrater agreement was not calculated. The codebook was then applied to all interviews, which were coded line-by-line.

After completion of the coding stage, comparison analysis [19] was conducted to uncover differences and similarities between "joiners" and "nonjoiners." To complete this analysis, we examined a number of important factors to determine if the groups differed on any of these factors. Factors examined included: if an injury had accompanied the fall, the stated cause of the fall (falling due to illness or medication verses falling due to environmental factors, such as slipping), aging-related loss, the effect of doctor referral, the emotional response to falling, participant core values, such as the importance of family and social connections, demographic factors, and program type (clinic versus homebased).

Atlas ti was used to perform both textual searches and visual displays of relationships and patterns emergent in the data. Initial themes resulting from this analysis were developed by one of the primary coders. An iterative process was then used to further develop and confirm the resultant themes by a third research team member.

\section{Results}

3.1. Recruitment. For the Fall Prevention Clinic, 35 letters of invitation were mailed to joiners and 40 to nonjoiners to reach our interview target of 20 completed interviews. For the EMS Fall Prevention Program, 30 letters were mailed to joiners and 37 to nonjoiners to reach our interview target of 20 completed interviews.

3.2. Demographics. Table 1 summarizes the demographics and self-perceived health of participants. Participants were predominantly females in their mid-70s. Just a few were nonwhite. Roughly one-quarter had an annual household income less than $\$ 15,000$. About half were college graduates. Most lived alone. There were no significant differences by joiner status on any of the sociodemographic characteristics that we examined.
Table 1: Participant demographics and self-perceived health, by joiner status.

\begin{tabular}{lcc}
\hline Characteristic & $\begin{array}{c}\text { Nonjoiner } \\
(N=20)\end{array}$ & $\begin{array}{c}\text { Joiner } \\
(N=19)\end{array}$ \\
\hline Female, \% & 83 & 70 \\
Age $\geq 75, \%$ & 30 & 45 \\
Age, years, mean (SD) & $76(11)$ & $77(10)$ \\
Married or partnered, \% & 22 & 25 \\
Non-white, \% & 6 & 0 \\
College graduate, \% & 44 & 47 \\
Household income $<\$ 15,000, \%$ & 29 & 26 \\
Lives alone, \% & 72 & 65 \\
Self-rated health excellent or very good, \% & 44 & 32 \\
\hline
\end{tabular}

3.3. Overarching Descriptive Themes. One of the most striking findings in this work was the similarities between joiners and nonjoiners of fall prevention programs. The two groups did not differ in most of the factors we examined. They were similar in their experience of loss associated with aging, in the core values they expressed, and in their emotional response to falling. In addition, we discovered that both groups of interviewees were using common public health terms in ways different from how these terms are typically used and understood by health professionals using the same terminology.

3.3.1. Loss Associated with Aging. The group as a whole expressed significant aging-related loss, both in their own physical abilities and in their familial or social relationships. Over $70 \%$ of participants expressed diminishing physical function, and over half expressed familial or social loss.

HFPC J5: I used to walk long distances every day, but I cannot do that anymore. I push it as far as I am comfortable pushing it. I regret that I cannot do it, but that's the way it is.

EMS J2: I have just dropped out of UW's courses that I was taking, because of a number of reasons, one of which was that it's so difficult for me to get around. Even though I have the services of Dial-a-Ride on campus, still in some cases, just getting to the DialA-Ride meeting place was very, very difficult.

EMS NJ4: Yes, I was married. My husband died on our 25th wedding anniversary so I have been alone ever since.

HFPC J6: Because in two-and-a-half years I lost my husband and five close relatives. It just has not left me in very good spirits most of the time, because I was close to all of my relatives. My brother was one of them, and it's just not been easy.

EMS NJ5: We have stopped doing lots of things that we did do, because it would take us longer to get to a meeting, for instance, and all of this kind of thing. 
3.3.2. Independence. Nearly all participants expressed the importance of independence and of being able to maintain their current level of functioning. Interestingly, this value was expressed regardless of their current circumstances and level of physical functioning.

EMS J7: I love my independence. I love it. I have never liked to depend on anyone.

HFPC J5: It (being independent) is very important. The wonderful thing about living here is that this is independent living, and although we have housekeeping and we have a dining room downstairs, we are free to come and go and do whatever we wish to do.

EMS NJ5: It's very important, because you just think that at least you can take care of yourself, if you cannot do all of these other things.

3.3.3. Emotional Responses to Falling. All participants in this study had experienced at least one fall, and many had sought medical attention for their fall. When asked how they felt right after the fall, the participants named emotional responses that were similar across groups, expressing anger at themselves, fear, and embarrassment.

HFPC J7: It was sort of like, "Boy, how clumsy can you get?" I didn't immediately start thinking that, "oh, no, I am not going to be able to take care of myself" or anything like that. I was sort of annoyed with myself that I had done that, more than anything else.

EMS NJ3: What have I done? I have really screwed up again. Then I said, "(Name), how could you have done this?"

HFPC NJ3: I was scared. I was mostly scared, because I said, "Oh, is this what life has for me?" I was falling so hard, and they tell us that you can break a hip. If you break a hip, you are on your way out. That just really was scary.

EMS J2: Well, you know, it's embarrassing. Here I am an old man, sort of, on the ground on my hands and knees.

In addition to the initial emotional response to falling, most participants expressed a heightened fear of falling and subsequent injury. They described trying to "be careful" to avoid falls and reported being very vigilant to avoid falling again.

HFPC NJ9: I try to be really careful, especially down my basement stairs. I mean, I have fallen there before, and I do not want that to happen again.

HFPC J7: I also watch where I put my feet now more often than I did before.

EMS NJ4: I am very careful. The rug, I watch because it wrinkles up, but I am very careful... Well, I do not want to break a hip.
3.4. Discrepancies in Terminology. As coding was being conducted and themes were developed, it became clear that the participant's understanding and usage of terms such as "independence" and "physically active" were not in line with how public health professionals typically use those terms. For example, participants considered themselves independent and valued that independence even when they were being cared for in a facility or by a caregiver. Their view of independence was much broader than the definitions used by health and public health professionals and focused on maintaining their current level of functioning.

Additionally, "physically active" was used by participants to refer to the activities of simple daily functioning; again a broader definition than is usually used in public health. When asked, "What first comes to mind when you think about being physically active, or staying physically fit?" A typical response was: "Well, being able to get out of bed, being able to dress myself, being able to feed myself, and being able to function in the way that most of us do or want to."

3.5. Barriers and Facilitators to Program Participation. The analysis of these interviews revealed just one primary difference between those who participated in one of the fall prevention programs and those who did not. Those who participated expressed that they "needed" the program; those who did not participate expressed a lack of need. Transportation was also cited as a barrier, although it was unclear if it was a primary barrier for those who cited it.

3.5.1. Perception of Need-Joiners. Those who joined a fall prevention program stated their need for the program in very simple terms, giving a sense that they would do everything they could to avoid falling again. When asked why they decided to go to the fall prevention clinic or have the in-home visit from EMS, 17 of the 20 interviewees responded with a simple variation of, "I saw the need."

HFPC J3: I needed to quit falling.

EMS J6: Well, I am most eager to learn anything that would be helpful for me.

3.5.2. Perception of Need-Nonjoiners. For those who chose not to participate, need perception also played a large role in two different ways. Half the group stated that they did not need the program because they felt they had not yet "reached the point" of needing intervention and that they could prevent falling themselves by being more careful.

EMS NJ10: I'd say, "Oh, I do not need that. I am just going to be aware." That's all that I can think of; I do not want that.

EMS NJ1: At this point in time in my life, I do not feel like I need the extensive help that I might need later.

Others interviewed felt there was "no need" for the program because they felt beyond help or did not believe that anything could be done to prevent falling. There was also a sense from these respondents that, having multiple health 
conditions, they felt overwhelmed thinking about one more intervention.

EMS NJ7: Well, I do not think that there's anything that you can do. I do not know what you can do.

HFPC NJ1: Yes, I am just not interested because I do not think that they can help me any, frankly.

HFPC NJ5: I have been through so many of these evaluations, and it's just yadda, yadda, yadda.

3.5.3. Transportation Barriers. Lack of transportation was the one other significant barrier that affected the Harborview Clinic participants. (EMS is an in-home program.) This barrier was discussed by 5 people who declined to participate in the Harborview clinic. This barrier was often mentioned in combination with other reasons for not participating, such as, "I just forgot," and "I am not sure what they do for me."

HFPC NJ2: I hate to ask people that I know to drive me, and so even from here to there, it's a bitch. That's my first thought, how am I going to get there? Then I put it out of my mind because I cannot go.

\section{Discussion}

The results of our study suggest that the main reasons for older adults accepting an invitation to participate in a fall risk assessment and management program relate to outcome expectations of the program. Participants who followed through on a referral to the fall prevention clinic or who received the home-based EMS fall risk assessment program believed that such a program would benefit them in helping them reduce their fall risk. Falling and its consequences instilled a great deal of anxiety and fear in our participants, and the older adults who chose to join a community fall prevention program reported interest in any program or resource that could help them prevent falls. In contrast, older adults who turned down the invitation for either program did not perceive a great need, either because they did not think they needed such a program at that particular point in their lives or because they felt they were beyond help. Nonjoiners reported relying on their own strategies (e.g., being more careful) for risk reduction.

These findings share some similarities with those reported in published studies involving elders in other countries. In a study of accepters and decliners of referral to a falls clinic in Denmark [13], all accepters believed that something could be done about their falls problem, that the time investment was worthwhile, and that they would use the advice received, whereas decliners expressed concerns that the healthcare system would take over their life, that the logistics and time commitment were excessive, and that providers had "hidden agendas" and might criticize them if they didn't follow advice. Yardley et al. [11] found that those they studied underestimated their personal susceptibility to falling and believed that no additional fall prevention measures were necessary. Those they interviewed also cited practical factors, such as transportation, effort, and cost as precluding their participation [11]. We did not identify among our sample the perception that fall prevention as a concept threatened personal identity and autonomy, as has been described by others [10]. However, in a departure from other published studies, we did find a prominent belief in self-reliance, that is, the power of the individual him- or herself to use their own methods to control his/her likelihood of falling again in the future. Of interest, a recent study of a sample $(N=36)$ of homebound women in their mid- $80 \mathrm{~s}$ to mid-90 s who resided in the Midwestern U.S. and had a history of falling in their home describes in great detail a variety of strategies that these women articulated as ways that they planned to avoid falling again, such as avoiding quick turns, taking care not to bend over, wearing shoes that will not slip, and using one's cane or walker more consistently [20].

In addition to perceived need and beliefs about the outcome of joining a fall risk assessment program, our participants highly valued "independence." However as mentioned above, participants' perception of independence was different from and more limited than what is usually presented in the literature. The same was true for other constructs, including "healthy aging" or "physical activity." That is, the predominant thoughts around independence, healthy aging, and physical activity were related to the ability to perform daily activities (i.e., get dressed by themselves, cook dinner, clean house). Participants took pride in listing the daily activities they were capable of doing (no matter how few), and their ability to maintain this level of "independence" was extremely important to them.

\subsection{Recommendations for Practice: Conveying Program Bene-} fits. The results of this qualitative study suggest that, in order to participate, older adults have to perceive a need for a community fall risk assessment program and that this perceived need is related to their assessment of how a program will benefit them in maintaining their current level of function. In portraying the benefits of a fall risk assessment program, it is important to emphasize program benefits that are consistent with the reality of many older adults' situation. Especially for high-risk elderly, who struggle with comorbidities, loss of physical abilities, and shrinking social networks, the emphasis should be on how falls are a major threat to remaining functional and independent $[2,3]$, and that through reducing the risk of falling by receiving and following recommendations provided by such preventive programs, these programs can help them maintain a level of confidence, mobility, and independence in their daily functioning [21].

\subsection{Recommendations for Practice: Desirable Program Char- acteristics. In addition, access to such programs needs to be simple. Assisting such older adults in getting to a clinic and back, perhaps through a "patient navigator" or a volunteer transportation program, may be instrumental in getting a high-risk population to a regional center that offers fall risk assessments. Engaging family caregiver support for an elder being counseled to undergo a fall risk assessment may also be helpful in encouraging participation. Regular, ongoing contact with a supportive health professional, such}


as a physical therapist, may increase motivation to continue fall prevention activities (e.g., doing routine balance and strength exercises) over time.

4.3. Recommendations for Practice: Advice from a Trusted Source. The results of this study are informative for healthcare practitioners and aging service providers seeking to engage high-risk elderly to participate in a fall risk assessment program. Having a history of having fallen did not necessarily lead participants to perceive that they needed to undergo an individualized risk assessment. This finding supports practitioners directly educating older persons about the fact that having experienced a fall suggests that one is highly likely to fall again in the future [22]. For the present generation of elderly, the advice of a personal physician has been shown to facilitate participation in fall prevention activities [12], and thus educational guidance, along with a recommendation to undergo a risk assessment, delivered by an informed primary care provider, may be invaluable in shaping an older adult's understanding of the importance of avoiding falls to well-being and the role of individualized risk assessment in this context.

With regard to limitations of the present study, one limitation is that only 1 out of every 4 eligible participants agreed to participate in this study, which may have resulted in a selection bias. Even so, we believe this selection bias is limited, since the total pool of eligible participants were fairly homogeneous due to the fact they had all been invited to undergo assessment and management by one of two fall prevention programs (which have their own eligibility criteria). A second limitation is that our study sample was not as racially diverse as we had anticipated; thus, further studies involving non-white persons are warranted.

Our study has several strengths. The sample was welldefined in terms of its sociodemographics, inclusive of those of low income, and uniform with respect to all participants being at high fall risk due to having had a history of at least one fall in the past. This latter point is important, in that it allowed us to circumvent the phenomenon of low perceived relevance of fall prevention due to not having experienced a prior fall [23]. In addition, we did not find major differences in attitudes between joiners and nonjoiners according to program. Participants who qualify for one program are typically eligible for the other program as well, and so this finding is perhaps not unexpected. The fact that findings for joiners and nonjoiners were similar regardless of program speaks to the external validity of our key results.

\section{Conclusions}

The study sample consisted of a group of elderly all of whom had experienced a fall. Many participants had experienced significant age-related losses. The perception of need was the critical factor in determining participation in the fall prevention programs offered. Interventions targeted at this high-risk group will need to address individual beliefs as well as structural and social factors (i.e., transportation issues, social networks) to optimize participation of this group in such programs.

\section{Acknowledgments}

The authors extend a heartfelt thanks to Jean Corr, physical therapist with the "One Step Ahead" program, for her invaluable assistance in identifying interview participants. This article was sponsored by the CDC Office of Public Health Research through its Centers of Excellence in Health Marketing and Health Communication program (Grant 5P01-CD000249-03). Additional funding support came from the University of Washington Health Promotion Research Center, one of CDC's Prevention Research Centers (HPRC cooperative agreement no. U48-DP-001911).

\section{References}

[1] M. E. Tinetti, M. Speechley, and S. F. Ginter, "Risk factors for falls among elderly persons living in the community," The New England Journal of Medicine, vol. 319, no. 26, pp. 1701-1707, 1988.

[2] M. E. Tinetti and C. S. Williams, "Falls, injuries due to falls, and the risk of admission to a nursing home," The New England Journal of Medicine, vol. 337, no. 18, pp. 1279-1284, 1997.

[3] M. E. Tinetti and C. S. Williams, "The effect of falls and fall injuries on functioning in community-dwelling older persons," The Journals of Gerontology: Series A, vol. 53, no. 2, pp. M112-M119, 1998.

[4] D. A. Sleet, D. B. Moffett, and J. Stevens, "CDC's research portfolio in older adult fall prevention: a review of progress, 1985-2005, and future research directions," Journal of Safety Research, vol. 39, no. 3, pp. 259-267, 2008.

[5] L. D. Gillespie, W. J. Gillespie, M. C. Robertson, S. E. Lamb, R. G. Cumming, and B. H. Rowe, "Interventions for preventing falls in elderly people," Cochrane Database of Systematic Reviews, no. 4, 2003.

[6] J. T. Chang, S. C. Morton, L. Z. Rubenstein et al., "Interventions for the prevention of falls in older adults: systematic review and meta-analysis of randomised clinical trials," British Medical Journal, vol. 328, no. 7441, pp. 680-683, 2004.

[7] M. E. Tinetti and C. Kumar, "The patient who falls: "It's always a trade-off'", Journal of the American Medical Association, vol. 303, no. 3, pp. 258-266, 2010.

[8] R. Boyd and J. A. Stevens, "Falls and fear of falling: burden, beliefs and behaviours," Age and Ageing, vol. 38, no. 4, pp. 423428, 2009.

[9] L. Yardley, S. Kirby, Y. Ben-Shlomo, R. Gilbert, S. Whitehead, and C. Todd, "How likely are older people to take up different falls prevention activities?" Preventive Medicine, vol. 47, no. 5 , pp. 554-558, 2008.

[10] L. Yardley, M. Donovan-Hall, K. Francis, and C. Todd, "Older people's views of advice about falls prevention: a qualitative study," Health Education Research, vol. 21, no. 4, pp. 508-517, 2006.

[11] L. Yardley, F. L. Bishop, N. Beyer et al., "Older people's views of falls-prevention interventions in six European countries," Gerontologist, vol. 46, no. 5, pp. 650-660, 2006.

[12] F. Bunn, A. Dickinson, E. Barnett-Page, E. Mcinnes, and K. Horton, "A systematic review of older people's perceptions of facilitators and barriers to participation in falls-prevention interventions," Ageing and Society, vol. 28, no. 4, pp. 449-472, 2008. 
[13] L. Evron, K. Schultz-Larsen, and T. Fristrup, "Barriers to participation in a hospital-based falls assessment clinic programme: an interview study with older people," Scandinavian Journal of Public Health, vol. 37, no. 7, pp. 728-735, 2009.

[14] World Health Organization, International Classification of Functioning, Disability and Health: ICF, World Health Organization, Geneva, Switzerland, 2001.

[15] M. B. Miles and A. M. Huberman, Qualitative Data Analysis: An Expanded Sourcebook, Sage Publications, Thousand Oaks, Calif, USA, 2nd edition, 1994.

[16] M. Moore, B. Williams, S. Ragsdale et al., "Translating a multifactorial fall prevention intervention into practice: a controlled evaluation of a fall prevention clinic," Journal of the American Geriatrics Society, vol. 58, no. 2, pp. 357-363, 2010.

[17] J. M. Corbin and A. Strauss, "Grounded theory research: procedures, canons, and evaluative criteria," Qualitative Sociology, vol. 13, no. 1, pp. 3-21, 1990.

[18] A. L. Strauss and J. M. Corbin, Basics of Qualitative Research: Techniques and Procedures for Developing Grounded Theory, Sage Publications, Thousand Oaks, Calif, USA, 2nd edition, 1998.

[19] B. G. Glaser and A. L. Strauss, The Discovery of Grounded Theory: Strategies for Qualitative Research, Aldine Press, New York, NY, USA, 1967.

[20] E. J. Porter, S. Matsuda, and E. J. Lindbloom, "Intentions of older homebound women to reduce the risk of falling again," Journal of Nursing Scholarship, vol. 42, no. 1, pp. 101-109, 2010.

[21] M. E. Tinetti, C. F. Mendes de Leon, J. T. Doucette, and D. I. Baker, "Fear of falling and fall-related efficacy in relationship to functioning among community-living elders," Journals of Gerontology, vol. 49, no. 3, pp. M140-M147, 1994.

[22] L. Z. Rubenstein and K. R. Josephson, "Falls and their prevention in elderly people: what does the evidence show?" Medical Clinics of North America, vol. 90, no. 5, pp. 807-824, 2006.

[23] E. McInnes and L. Askie, "Evidence review on older people's views and experiences of falls prevention strategies," Worldviews on Evidence-Based Nursing, vol. 1, no. 1, pp. 20-37, 2004. 


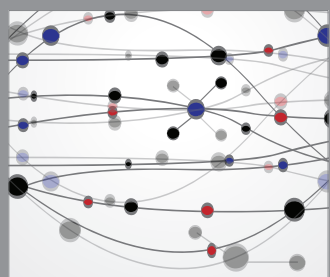

The Scientific World Journal
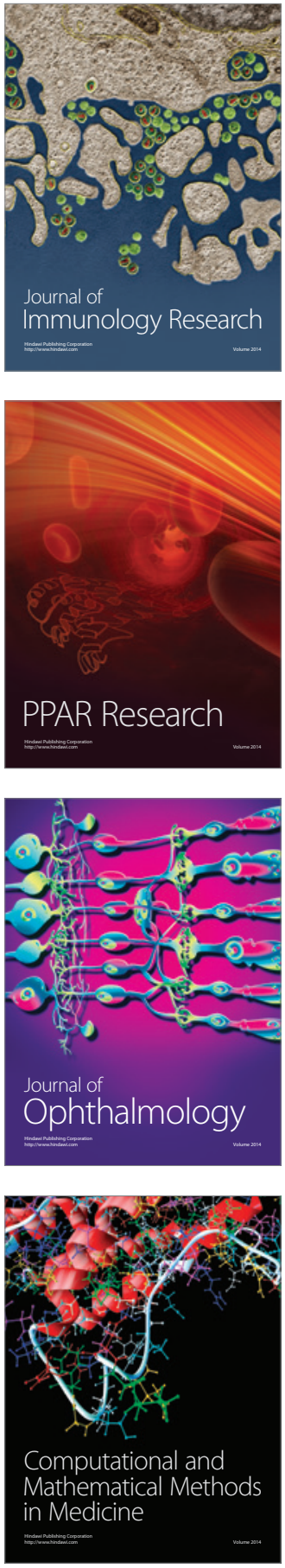

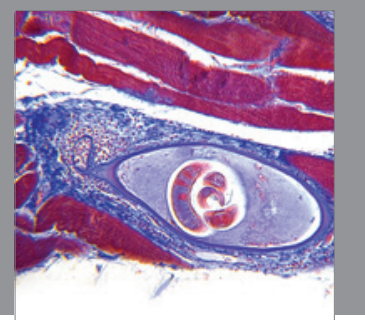

Gastroenterology

Research and Practice
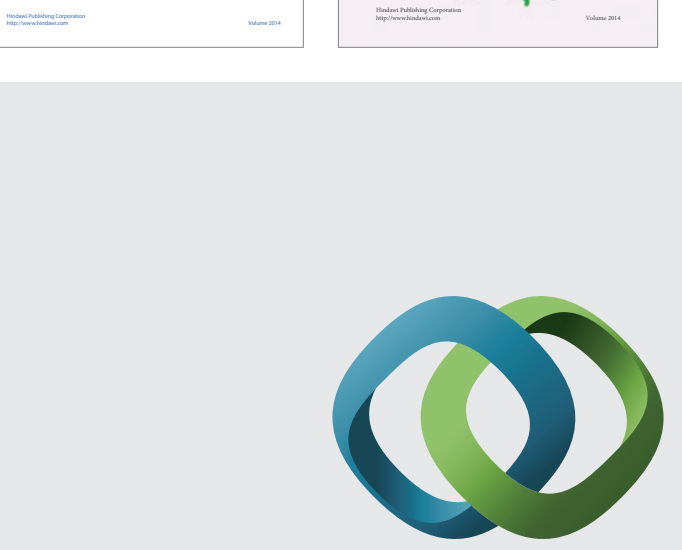

\section{Hindawi}

Submit your manuscripts at

http://www.hindawi.com
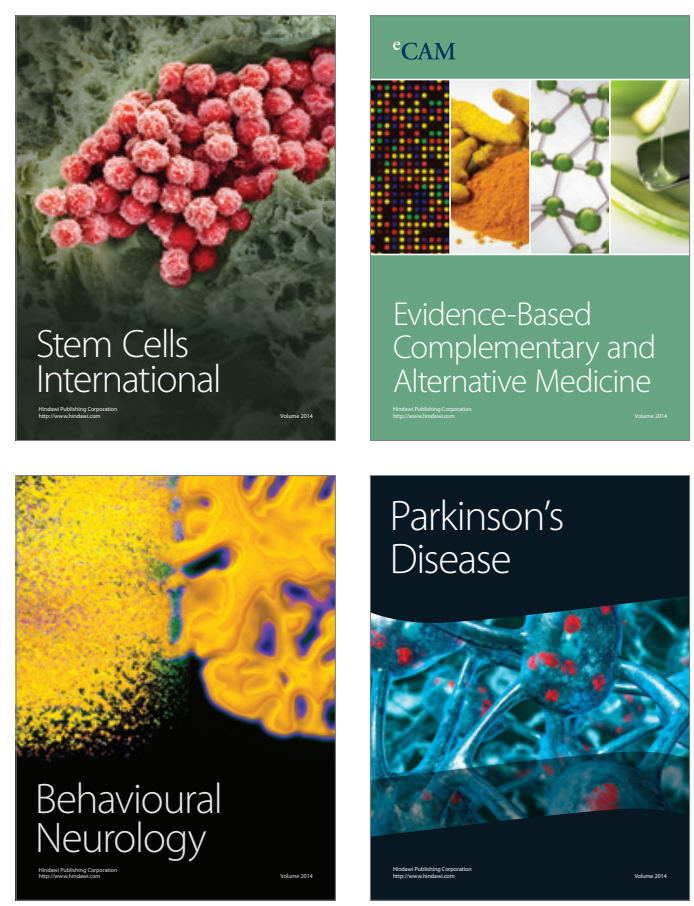

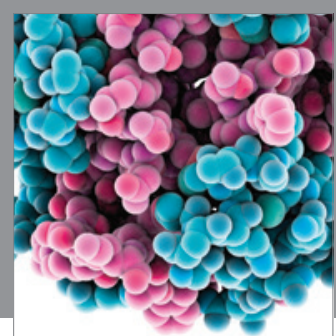

Journal of
Diabetes Research

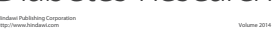

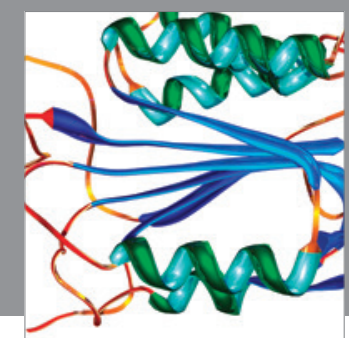

Disease Markers
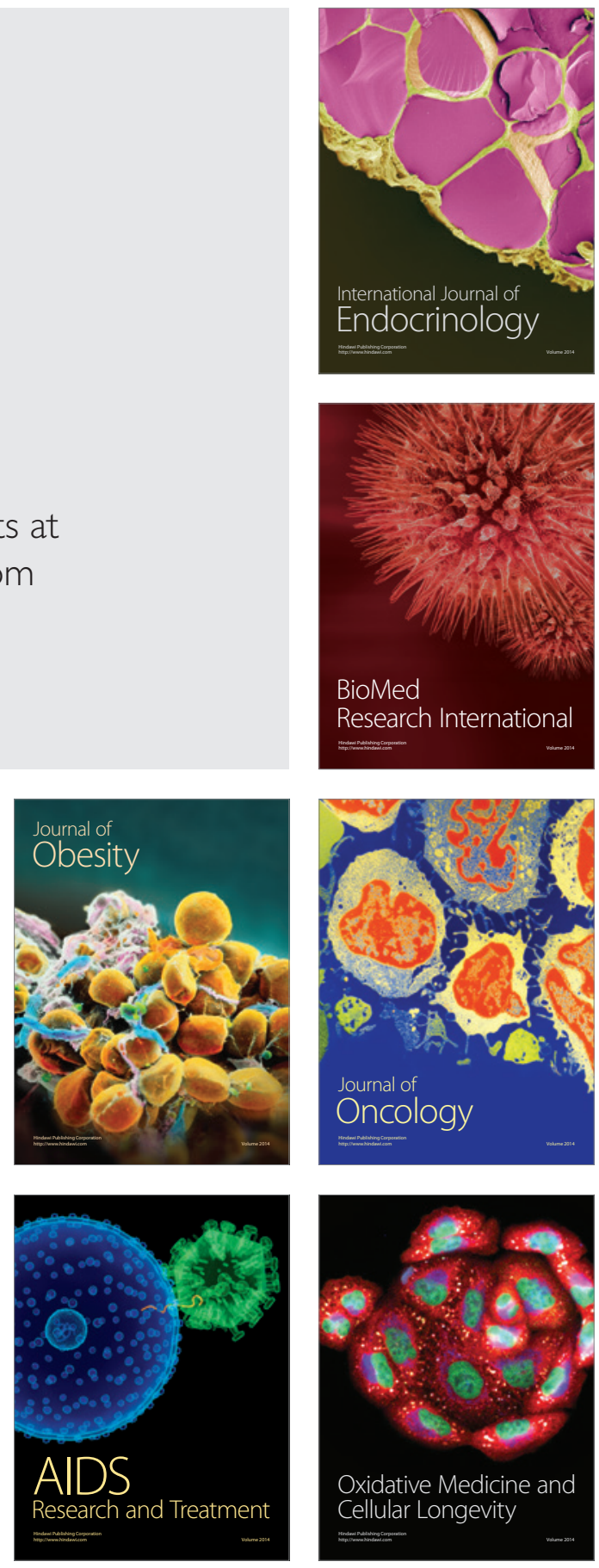